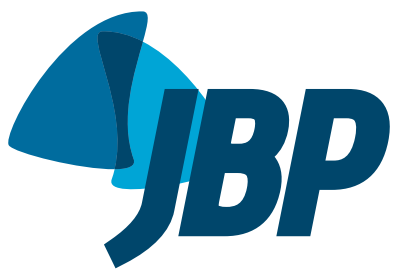

\title{
Video-assisted thoracoscopic thoracic duct ligation with near-infrared fluorescence imaging with indocyanine green
}

\author{
Benoit Jacques Bibas ${ }^{1, a}$, Rafael Lucas Costa-de-Carvalho ${ }^{1, b}$, \\ Flavio Pola-dos-Reis ${ }^{1, c}$, Leticia Leone Lauricella ${ }^{1, d}$, \\ Paulo Manoel Pêgo-Fernandes ${ }^{1, e}$, Ricardo Mingarini Terra ${ }^{1, f}$
}

\section{TO THE EDITOR:}

Surgical ligation of the thoracic duct can be a challenging procedure. If a chyle leak can be identified, direct ligation with nonabsorbable suture should be performed. ${ }^{(1)}$ In cases in which the chyle leak or the duct itself cannot be identified, mass ligation of the duct performed above the esophageal hiatus is the approach of choice. (2) This ensures duct ligation at its entry in the chest, thus sealing all of the accessory ducts that could be the source of the chylothorax. $(2,3)$ Thoracic duct ligation (TDL) is typically performed by means of thoracoscopy, which allows image magnification and facilitates identification of the site of chyle leak. ${ }^{(1)}$ Enteral administration of a fat source such as olive oil or cream during the procedure can be used in order to assist in locating the site of leak by increasing the flow of chyle. ${ }^{(1)}$ Invisible near-infrared (NIR) fluorescence imaging with indocyanine green (ICG) is a new imaging modality that combines NIR imaging and a fluorescent dye (ICG) to improve visualization during surgery. NIR fluorescence imaging with ICG allows visualization of the light emitted from the dye, which is then superimposed onto the video image, thus highlighting the fluorescence site. ${ }^{(4)}$ Below, we describe the case of a patient in whom NIR fluorescence imaging with ICG was used.

A 55-year-old male patient was diagnosed with oropharyngeal squamous cell carcinoma with left cervical lymph node involvement. Six months after having undergone treatment with carboplatin and paclitaxel followed by radiation therapy (70 Gy), the patient experienced lymph node disease recurrence. Radical neck dissection was therefore performed. Three days after the procedure, a chest X-ray showed pleural effusion. The effusion was sampled. Pleural fluid analysis showed elevated triglyceride levels (of $450 \mathrm{mg} / \mathrm{dL}$ ), which were consistent with a chylothorax. Pleural drainage was therefore performed. On the same day, fluid draining from the cervical wound was analyzed and found to be consistent with chyle. The patient was started on a regular medium-chain triglyceride diet and responded well to treatment, as evidenced by a reduction in fluid output from the chest tube and the cervical drain, both of which were removed four days later. The patient was discharged two days after the chest tube and the cervical drain had been removed. One week later, he returned to the emergency room with a neck lump (Figures $1 \mathrm{~A}, \mathrm{~B}$, and C). A chest X-ray was normal. Percutaneous catheter drainage was performed. The fluid had a milky appearance and elevated triglyceride levels (of $350 \mathrm{mg} / \mathrm{dL}$ ). Because management with oral fasting, parenteral nutrition, and octreotide therapy for 15 days failed, a decision was made to perform a TDL. For better visualization of the thoracic duct, $50 \mathrm{~mL}$ of olive oil were administered enterally 60 min before the procedure. Under general anesthesia, the patient underwent three-port video-assisted thoracoscopic TDL with ICG-assisted NIR fluorescence imaging, a PINPOINT ${ }^{\circledR}$ endoscopic fluorescence imaging system (Stryker Corporation, Kalamazoo, MI, USA) being used. With the use of ultrasound to identify inguinal lymph nodes, $2 \mathrm{~mL}$ of $0.5 \%$ ICG solution were injected bilaterally. Approximately 5 min after local massage, the thoracic duct was visible as green fluorescence, no chyle leak being present (Figure 1D). Metal clips were used for TDL (Figure 1E). The patient made an uncomplicated recovery. The chest tube was removed two days after the procedure, and the patient was discharged four days after the procedure. There was no recurrence of the chylous fistula.

ICG is a disulfonated heptamethine indocyanine small molecule that has been approved by the US Food and Drug Administration for cardiac output monitoring, liver function testing, hepatic blood flow measurement, and ophthalmic angiography. ${ }^{(5)}$ ICG binds to plasma lipoproteins and is activated by NIR rays of 760-780 $\mathrm{nm}$, resulting in fluorescence. It has an extremely small hydrodynamic diameter, which allows it to travel through blood vessels, lymph nodes, and lymphatic ducts. $^{(5)}$ The development of equipment that allows fluorescent images to be superimposed onto video images in real time allowed ICG to be used in many surgical applications, including evaluation of flap perfusion, evaluation of anastomotic perfusion, identification of anatomical structures (such as blood vessels, biliary vessels, and lymphatic vessels), and identification of lymphatic drainage in cancer surgery. ${ }^{(6,7)}$ In thoracic surgery, NIR fluorescence imaging with ICG can be used for identification of lung nodules, identification of the intersegmental plane, and conduit vascular evaluation during esophagectomy. ${ }^{(4)}$ According to Ashitate et al., ${ }^{(5)}$ injection of ICG into the lower leg of pigs provided thoracic duct imaging with an onset of approximately 5 min after injection, as well as sustained imaging for at least $60 \mathrm{~min}$ after injection. In addition, an injury 
model showed that it is possible to identify chyle leaks without loss of accuracy. ${ }^{(5)}$

The use of ICG for the clinical treatment of chylothorax is still relatively new. ${ }^{(8,9)}$ Chang et al. ${ }^{(9)}$ reported the case of a three-month-old infant with congenital heart disease and postoperative chylothorax. During repeat sternotomy, no chyle leak was found. NIR fluorescence imaging with ICG allowed visualization and treatment of a fistula in an unusual location (i.e., lateral to the aorta). Kaburagi et al.( ${ }^{(8)}$ described the case of a patient in whom ICG was injected into the mesentery during transabdominal TDL for the management of postesophagectomy chylothorax.
In the case reported here, NIR fluorescence imaging with ICG was used in order to identify the thoracic duct, improving its visualization. The need for injecting ICG into lymph nodes represented a technical challenge, which was overcome with the use of ultrasound-guided injection. NIR fluorescence imaging with ICG provides excellent real-time intraoperative visualization of the thoracic duct, being particularly useful in cases in which identification of the thoracic duct or chylous fistula is likely to be difficult, including reoperations, procedures performed after radiation therapy, and procedures performed in children, as well as cases in which there is no active chyle leak in the chest.
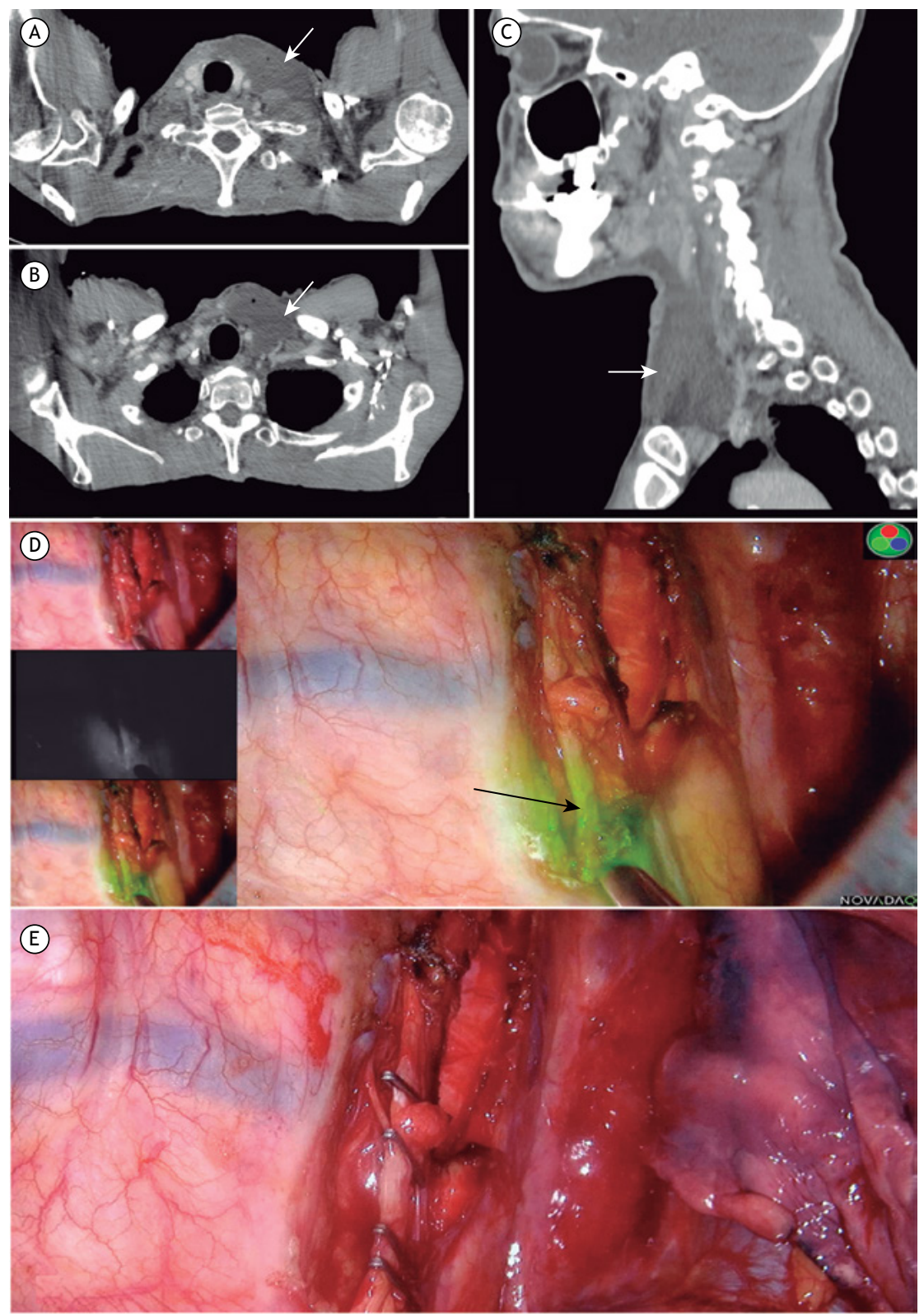

Figure 1. In A and B, CT scan of the neck showing a large fluid collection (arrow). In C, sagittal CT reconstruction. In $D$, intraoperative view of the thoracic duct dissected after injection of indocyanine green (arrow). In $E$, thoracic duct ligation with metal clips. 


\section{REFERENCES}

1. Nair SK, Petko M, Hayward MP. Aetiology and management of chylothorax in adults. Eur J Cardiothoracic Surg. 2007;32(2):362-9. https://doi.org/10.1016/j.ejcts.2007.04.024

2. Martucci N, Tracey M, Rocco G. Postoperative Chylothorax. Thorac Surg Clin. 2015;25(4):523-8. https://doi.org/10.1016/j. thorsurg.2015.07.014

3. Patterson GA, Todd TR, Delarue NC, Ilves R, Pearson FG, Cooper JD. Supradiaphragmatic ligation of the thoracic duct in intractable chylous fistula. Ann Thorac Surg. 1981;32(1):44-9. https://doi.org/10.1016/ S0003-4975(10)61372-0

4. Chiu C, Chao Y, Liu Y, Wen C, Chen W, Wu C, et al. Clinical use of near-infrared fluorescence imaging with indocyanine green in thoracic surgery: a literature review. J Thorac Dis. 2016;8(Suppl 9):S744-S748. https://doi.org/10.21037/jtd.2016.09.70

5. Ashitate Y, Tanaka E, Stockdale A, Choi S, Frangioni JV. Near-infrared fluorescence imaging of thoracic duct anatomy and function in open surgery and video-assisted thoracic surgery. J Thorac Cardiovasc Surg. 2011;142(1):31-8.e1-2. https://doi.org/10.1016/j.jtcvs.2011.03.004

6. Spinoglio G, Bertani E, Borin S, Piccioli A, Petz W. Green indocyanine fluorescence in robotic abdominal surgery. Updates Surg. 2018;70(3):375-379. https://doi.org/10.1007/s13304-018-0585-6

7. Baiocchi GL, Diana M, Boni L. Indocyanine green-based fluorescence imaging in visceral and hepatobiliary and pancreatic surgery: State of the art and future directions. World J Gastroenterol. 2018;24(27):2921-2930. https://doi.org/10.3748/wjg.v24.i27.2921

8. Kaburagi T, Takeuchi H, Oyama T, Nakamura R, Takahashi T, Wada $\mathrm{N}$, et al. Intraoperative fluorescence lymphography using indocyanine green in a patient with chylothorax after esophagectomy : report of a case. Surg Today. 2013:43(2):206-10. https://doi.org/10.1007/ s00595-012-0391-6

9. Chang $\mathrm{TI}$, Chen YS, Huang SC. Intraoperative indocyanine green fluorescence lymphography to detect chylous leakage sites after congenital heart surgery. J Thorac Cardiovasc Surg. 2014;148(2):739 40. https://doi.org/10.1016/j.jtcvs.2014.03.021 